\title{
Excitation spectra of strongly correlated lattice bosons and polaritons
}

\author{
Peter Pippan, ${ }^{1}$ Hans Gerd Evertz, ${ }^{1}$ and Martin Hohenadler ${ }^{2}$, $*$ \\ ${ }^{I}$ Institute for Theoretical and Computational Physics, TU Graz, 8010 Graz, Austria \\ ${ }^{2}$ Cavendish Laboratory, University of Cambridge, Cambridge, CB3 OHE, United Kingdom
}

(Dated: October 24, 2018)

\begin{abstract}
Spectral properties of the Bose-Hubbard model and a recently proposed coupled-cavity model are studied by means of quantum Monte Carlo simulations in one dimension. Both models exhibit a quantum phase transition from a Mott insulator to a superfluid phase. The dynamic structure factor $S(k, \omega)$ and the single-particle spectrum $A(k, \omega)$ are calculated, focusing on the parameter region around the phase transition from the Mott insulator with density one to the superfluid phase, where correlations are important. The strongly interacting nature of the superfluid phase manifests itself in terms of additional gapped modes in the spectra. Comparison is made to recent analytical work on the Bose-Hubbard model. Despite some subtle differences due to the polaritonic particles in the cavity model, the gross features are found to be very similar to the Bose-Hubbard case. For the polariton model, emergent particle-hole symmetry near the Mott lobe tip is demonstrated, and temperature and detuning effects are analyzed. A scaling analysis for the generic transition suggests mean field exponents, in accordance with field theory results.
\end{abstract}

PACS numbers: 67.25.D-,67.25.dj,67.25.dt,64.70.Tg,71.36.+c,42.50.Ct,03.75.Kk

\section{INTRODUCTION}

The Mott insulator (MI) to superfluid (SF) quantum phase transition in the generic Bose-Hubbard model ${ }^{1}$ has attracted a lot of attention in recent years due to the progress in experiments on cold atomic gases in optical lattices.$^{2}$ More recently, there have also been significant advances in the coherent coupling of single atoms and cold atomic gases to cavity radiation (cavity quantum electrodynamics) ${ }^{3 / 4}$ A clean realization of the Jaynes-Cummings Hamiltonian has been achieved by coupling a superconducting qubit to a microwave cavity ${ }^{5}$ On the theory side, multi-component Bose gases coupled to light have e.g. been shown to support a superradiant Mott insulator phase with polariton condensation. 6

In parallel, several theoretical proposals have shown the possibility of having a state of strongly correlated photons or polaritons in solid-state systems of coupled cavity arrays (also referred to as polariton models or Jaynes-CummingsHubbard models), ${ }^{778}$ and a review of work along these lines has been given ${ }^{9}$ The possibility of preparing a system of photons in a Mott state with one photon per site is a promising starting point for quantum information processing. An important feature shared with cold atomic gases coupled to light is the composite nature of the polaritons. Particularly attractive properties of cavity arrays would include accessibility of local properties in measurements and scalability in terms of size. Perhaps the most likely candidate for setting up such a model experimentally is based on extending the work on superconducting qubits to arrays: ${ }^{510}$ In contrast to cold atomic gases, where the interaction and/or hopping strength can be varied, the phase transition may be observed by changing the detuning between the two-level system and the resonator. Analysis of coupled cavity models is fruitful in its own right, as a detailed understanding of the corresponding models offers insight into strongly correlated polariton systems. An important aspect of such studies is the extent to which such systems resemble the familiar Bose-Hubbard physics.

From the above examples and many more in the litera- ture, it is apparent that interacting boson systems on a lattice are of great interest for the progress of both theory and experiment. Compared to Bose fluids, the lattice changes the physics in several aspects. Although long-range phase coherence still gives rise to phonon excitations-despite the breaking of translational symmetry - the quenching of the kinetic energy makes the system much more strongly correlated ${ }^{11}$ Besides, the lattice allows the formation of incompressible MI states with the same integer particle number at each site.

A large amount of work has been devoted to detailed studies of the Bose-Hubbard model, leading to a wealth of knowledge with and without additional complications such as trapping potentials or disorder. However, the dynamical properties and excitations in particular of the SF phase in the vicinity of the quantum phase transition, are still not completely understood. A number of authors have addressed the dynamics of the Bose-Hubbard model in different dimensions, $12|13| 14|15| 16|17| 18|1922021| 22 \mid 23]$ with results providing valuable information about the underlying physics, while corresponding work on coupled cavity models has just begun. $24 / 25$ The two most important dynamic observables are the dynamic structure factor and the single-particle spectral function, which are also at the heart of theoretical and experimental works on Bose fluids. ${ }^{26}$ Experimentally, the dynamic structure factor may be measured by Bragg spectroscopy or lattice modulation (in cold atomic gases) as well as by neutron scattering (in liquid helium), and single-particle excitations of optical solid-state systems are accessible by means of photoluminescence measurements.

Whereas the standard Bose-Hubbard model only supports MI and SF phases, the physics of the polariton models is slightly richer. Owing to the composite nature of the conserved particles (polaritons), these phases can either be of polaritonic, excitonic or photonic character ${ }^{24(2728129}$ with distinct dynamic properties. Which of the cases is realized depends on the value of the detuning between the cavity mode and the transition frequency of the atoms that mediate polariton repulsion. Very recently it has been proposed that the fractional 
quantum Hall effect may also be realized in coupled cavity arrays .30

In general, accurate and unbiased results are very hard to obtain. Most existing work on spectral properties in the Mott phase is based on mean-field and/or strong-coupling approximations, in which fluctuations of the particle numbers are more or less restricted. Results of extensive strong coupling expansions for the phase diagram ${ }^{13] 31} \mathrm{do}$, however, agree very well with precise density-matrix renormalization group (DMRG) ${ }^{14}$ and quantum Monte Carlo (QMC) results. ${ }^{2021}$ Bogoliubov type descriptions have been found to accurately describe the SF phase only in the limit of weak interaction, and fail to account for the transition to a MI and correlation features in the SF close to the transition. Hence the most interesting (and most difficult) regime is that near the quantum phase transition, where quantum fluctuations and correlation effects cannot be neglected.

In one dimension (1D), quantum fluctuation effects are particularly pronounced and mean-field methods are in general insufficient. Notable exceptions include situations where coupling to additional degrees of freedom provides an effective long-range interaction! $\mathrm{An}$ interesting aspect of $1 \mathrm{D}$ is that for strong (repulsive) interaction, fermions and bosons behave in a very similar way, and that the low-energy, long-wavelength physics is described by the Luttinger liquid model ${ }^{32}$

In the present paper we employ the directed loop quantum Monte Carlo method, ${ }^{33}$ which is exact and therefore yields unbiased results also in difficult parameter regimes. Importantly, our simulations preserve the full quantum dynamics.

Few nonperturbative results are available for the spectra in the Bose-Hubbard model, namely for the dynamical conductivity,,$\frac{14}{4}$ for the dynamic structure factor $S(k, \omega) \sqrt{15 \mid 16}$ on small systems, and for the single-boson spectral function $A(k, \omega)$ in the Mott phase deduced from small systems, $\frac{18}{18}$ all in 1D. For the polariton model considered here, only $A(k, \omega)$ in the Mott phase has been calculated. ${ }^{24}$

The focus of our work is therefore on the calculation of excitation spectra for both the Bose-Hubbard model and the polariton model within and around the first Mott lobe (i.e., the lobe with density one), for which comparison to recent analytical and numerical results is made. Other issues addressed include the sound velocity in the SF phase, particle and hole masses, as well as temperature and detuning effects for the case of the polariton model.

Our simulations are performed at low but finite temperatures. On one hand, this complicates the analysis of the results, but on the other hand it matches the experimental situation.26134

The paper is organized as follows. In Sec. II we introduce the two models considered. Section III contains some details about the method. Results are discussed in Sec. IV, and in Sec. $\nabla$ we present our conclusions.

\section{MODELS}

The polariton model we consider is the simplest among several recent proposals. ${ }^{[789135136}$ It describes an array of $L$ optical microcavities, each of which contains a single two-level atom with states $|\downarrow\rangle,|\uparrow\rangle$ separated by energy $\epsilon$. Within the rotating wave approximation one such cavity is represented by the Jaynes-Cummings Hamiltonian $\frac{37}{}(\hbar=1)$

$$
\begin{aligned}
\hat{H}_{i}^{\mathrm{JC}}= & \epsilon\left|\uparrow_{i}\right\rangle\left\langle\uparrow_{i}\right|+\omega_{0} a_{i}^{\dagger} a_{i} \\
& +g\left(\left|\uparrow_{i}\right\rangle\left\langle\downarrow_{i}\left|a_{i}+\right| \downarrow_{i}\right\rangle\left\langle\uparrow_{i}\right| a_{i}^{\dagger}\right) .
\end{aligned}
$$

Here $\omega_{0}$ is the cavity photon energy, and $\Delta=\epsilon-\omega_{0}$ defines the detuning. The atom-photon coupling $g\left(a_{i}^{\dagger}, a_{i}\right.$ are photon creation and annihilation operators) gives rise to formation of polaritons (combined atom-photon or exciton-photon excitations). Allowing for nearest-neighbor photon hopping between cavities with amplitude $t$ leads to the lattice Hamiltonian

$$
\hat{H}^{\mathrm{PM}}=-t \sum_{\langle i, j\rangle} a_{i}^{\dagger} a_{j}+\sum_{i} \hat{H}_{i}^{\mathrm{JC}}-\mu \hat{N}_{\mathrm{p}} .
$$

The conserved polariton number $\hat{N}_{\mathrm{p}}=\sum_{i} \hat{n}_{\mathrm{p}, i}$, with $\hat{n}_{\mathrm{p}, i}=$ $a_{i}^{\dagger} a_{i}+\left|\uparrow_{i}\right\rangle\left\langle\uparrow_{i}\right|$, is determined by the chemical potential

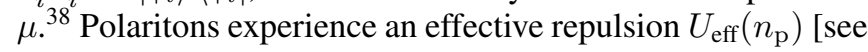
Eq. [11]] due to the nonlinear dependence of the single-site energy on the local occupation number $n_{\mathrm{p}}$. We use $g$ as the unit of energy and set $\omega_{0} / g, k_{\mathrm{B}}$ and the lattice constant equal to unity. The rotating wave approximation becomes unjustified for $g$ comparable to $\epsilon$. The motivation for setting $g=\epsilon$ is direct comparison to previous work. The Hamiltonian (2) has been studied in [7|9|10|24|25|27|28|29|35|38|39|40].

We also consider the Bose-Hubbard Hamiltonian

$$
\hat{H}^{\mathrm{BHM}}=-t \sum_{\langle i, j\rangle} b_{i}^{\dagger} b_{j}+\frac{U}{2} \sum_{i} n_{i}\left(n_{i}-1\right)-\mu \hat{N},
$$

describing soft-core bosons with repulsion $U$ and hopping $t$. Here $\hat{N}=\sum_{i} \hat{n}_{i}=\sum_{i} b_{i}^{\dagger} b_{i}$, is the total number of bosons, and we use $U$ as the unit of energy.

As an alternative to the spin language used here, the polariton model (2) can be written as a two-band Bose-Hubbard Hamiltonian, $4 \Gamma$ one boson species is itinerant, whereas the other is immobile (corresponding to localized excitons) with a hard-core constraint. This correspondence provides a direct connection to recent work on cold atomic gases in optical lattices, with the natural extension to the case where the excitons are mobile as well. ${ }^{6}$

We shall see below that owing to the composite nature of the bosonic particles in the polariton model, it is generally easier to understand the features of the Bose-Hubbard model first, and then explore similarities to the polariton model. Moreover, analytical approximations are more readily available for the Bose-Hubbard model and provide insight into the numerical data. Periodic boundary conditions in real space are applied in all simulations, and the system size is denoted as $L$.

\section{METHOD}

We use the directed loop method, $\frac{33}{3}$ a generalization of the loop algorithm,,$\sqrt{4243}$ which has no systematic errors and is efficient (low autocorrelations), facilitating the simulation of 
large systems at low temperatures. We make use of the ALPS library $\sqrt{4445}$ and of the ALPS applications, ${ }^{46}$ which use the stochastic series expansion (SSE) representation ${ }^{47}$ of worldline path integrals. We have verified that we obtain the correct phase boundary in 1D for selected points in parameter space.

In contrast to most previous QMC calculations of the BoseHubbard model, the focus of the current paper is on dynamical properties. The SSE representation has the drawback that dynamical correlation functions in imaginary time, which we need to obtain spectra, are very inefficient to calculate, since they involve a convolution of Green functions at different SSE distances ${ }^{48}$ On the other hand, Green functions can be measured easily in an imaginary time representation. For this reason we revert a mapping from continuous time to $\mathrm{SSE}^{49}$ when measuring Green functions. To each operator in a given SSE operator string we associate a time $\tau \in[0, \beta]$ which is stochastically sampled out of a uniform distribution. This maps the SSE configuration into a worldline configuration in continuous imaginary time ${ }^{50}$

Correlation functions of diagonal operators can then be measured directly. For example, in the case of $\left\langle\hat{\rho}_{i}(\tau) \hat{\rho}_{j}(0)\right\rangle$ we evaluate the density $\rho_{i}(\tau)$ on a fine time grid. This time discretization limits the high energy range of the Green function, but does not introduce any discretization error to the QMC algorithm itself. With the Fourier transformation of the density $\mathcal{F}\left(\hat{\rho}_{i}(\tau)\right)=\hat{\rho}_{k, \omega}$, we measure the correlation function $\mathcal{F}\left(\left\langle\hat{\rho}_{i}(\tau) \hat{\rho}_{j}(0)\right\rangle\right)=\left\langle\hat{\rho}_{k, \omega} \hat{\rho}_{-k, \omega}\right\rangle$ using fast Fourier transforms.

The evaluation of off-diagonal single-particle correlation functions of the form $\left\langle\psi_{i}(\tau) \psi_{0}^{\dagger}(0)\right\rangle$ requires some care. We again make use of the worldline picture, in which two operators $\psi^{\dagger}$ and $\psi$ are inserted whenever a new loop update starts. Let us assume that $\psi$ moves around (loop head) while $\psi^{\dagger}$ is pinned (loop tail). The time and position of the loop tail are set as the new origin of our coordinate system, and we store the values $\left\langle\alpha\left|\psi_{i}(\tau) \psi_{0}^{\dagger}(0)\right| \beta\right\rangle$ whenever the loop head $\psi_{i}(\tau)$ crosses a point on the time grid with distance $(i, \tau)$ from the new origin. Here $|\alpha\rangle,|\beta\rangle$ are the states in the world line configuration prior to the arrival of the loop head. We then again use fast Fourier transformation to evaluate the correlation functions in Fourier space.

Let us now define the observables of interest. The quantum phase transition can be detected by calculating the superfluid density $\rho_{\mathrm{s}}$, measured in the simulations in terms of the spatial winding number $w$ as $\rho_{\mathrm{s}}=L\left\langle w^{2}\right\rangle / \beta,{ }^{[152]} \beta=1 / k T$ being the inverse temperature. Another important observable in the context of the MI-SF transition is the total density, $n=\langle\hat{N}\rangle / L$ in the Bose-Hubbard model, and $n_{\mathrm{p}}=\left\langle\hat{N}_{\mathrm{p}}\right\rangle / L$ in the polariton model.

Concerning dynamical properties, we compute the dynamic structure factor $S(k, \omega)$ and the single-particle spectral function $A(k, \omega)$. The dynamic structure factor at momentum $k$ and energy $\omega$ is given by

$$
\begin{aligned}
S(k, \omega) & =\frac{1}{2 \pi L} \int_{-\infty}^{\infty} d \tau e^{\mathrm{i} \omega \tau}\left\langle\hat{\rho}_{k}(\tau) \hat{\rho}_{k}^{\dagger}(0)\right\rangle \\
& =\frac{1}{L} \sum_{n, m} \frac{e^{-\beta E_{n}}}{Z}\left|\left\langle m\left|\hat{\rho}_{k}^{\dagger}\right| n\right\rangle\right|^{2} \delta\left[\omega-\left(E_{m}-E_{n}\right)\right],
\end{aligned}
$$

with the grand-canonical partition function $Z$ and the energy of the $n$th eigenstate $E_{n}$. In our simulations, $S(k, \omega)$ is obtained from

$$
\left\langle\hat{\rho}_{k}(\tau) \hat{\rho}_{-k}(0)\right\rangle=\int d \omega S(k, \omega) \frac{e^{-\tau \omega}}{1+e^{-\omega \beta}}
$$

by means of the maximum entropy method.

For the Bose-Hubbard model, the density operator $\hat{\rho}_{i}=$ $\hat{n}_{i}$, and $\rho_{k}^{\dagger}=\sum_{q} b_{q+k}^{\dagger} b_{q}$. For the polariton model, we can calculate the dynamic structure factor for photons $\left[S^{\mathrm{ph}}(k, \omega)\right]$, atoms $\left[S^{\text {at }}(k, \omega)\right]$ or polaritons $[S(k, \omega)]$ by using

$$
\hat{\rho}_{i}= \begin{cases}a_{i}^{\dagger} a_{i} & \text { for photons, } \\ \left|\uparrow_{i}\right\rangle\left\langle\uparrow_{i}\right| & \text { for atoms, } \\ a_{i}^{\dagger} a_{i}+\left|\uparrow_{i}\right\rangle\left\langle\uparrow_{i}\right| & \text { for polaritons, }\end{cases}
$$

respectively.

The single-particle spectral function is defined as

$$
\begin{aligned}
A(k, \omega) & =-\frac{1}{\pi} \operatorname{Im}\left\langle\left\langle\hat{\psi}_{k} ; \hat{\psi}_{k}^{\dagger}\right\rangle\right\rangle_{\omega} \\
& =\sum_{n, m} \frac{e^{-\beta E_{n}}}{Z}\left|\left\langle m\left|\hat{\psi}_{k}^{\dagger}\right| n\right\rangle\right|^{2} \delta\left[\omega-\left(E_{m}-E_{n}\right)\right],
\end{aligned}
$$

where the real-space operator $\hat{\psi}_{i}$ entering the Green function is given by $\hat{\psi}_{i}=b_{i}$ for the Bose-Hubbard model, and by $\hat{\psi}_{i}=$ $a_{i}$ for the polariton model. maximum entropy is again used to map to real frequencies.

The QMC algorithm samples the partition function in the grand canonical ensemble. However, using only those configurations which have a given number of polaritons enables us to measure observables in the canonical ensemble as well. Here this simple but powerful trick permits us to study the fixed-density phase transition which occurs in the polariton model as a function of $t / \mathrm{g}$.

The SSE representation requires to set a maximum boson number per site. In the Bose-Hubbard model, we allow a maximum of six bosons per site. In the polariton model we allow from six (Mott insulator, fixed-density transition) up to 16 (SF phase) photons per site. Convergence has been monitored by plotting histograms of the photon number distribution, and the cut-offs have been chosen generously such that there was no truncation error.

\section{RESULTS}

We begin with a review of the properties of the BoseHubbard model and the polariton model as they emerge 
from previous work. Whereas a substantial literature exists for the Bose-Hubbard model, work on the polariton model began only recently, based on mean-field theory, ${ }^{7 / 10}$ exact diagonalization,, 38 the DMRG,, 39 the variational cluster approach, 24, QMC 53 and strong coupling theory ${ }^{[25}$ Our discussion focuses on 1D, and follows Fisher et al! $!^{1}$ and Kühner et al. 14

The Bose-Hubbard model describes the competition of kinetic energy and local, repulsive interaction. Depending on the ratio $t / U$ and the density of bosons $n$ (the system is superfluid for any $t>0$ if $n$ is not integer), the Bose-Hubbard model at temperature $T=0$ is either in a MI state or in a SF state. The MI is characterized by an integer particle density, phase fluctuations and a gap in the single-particle excitation spectrum. In the SF phase, we have significant density fluctuations, phase coherence, and nonzero superfluid density $\rho_{\mathrm{s}}$, as well as gapless (phonon) excitations with linear dispersion at small $k$.

For the case of one dimension considered here, a precise zero-temperature phase diagram in the $\mu / U, t / U$ plane has been determined by Kühner et al., 14 and these data are shown in Fig. 1.a). There exists a Mott lobe inside which the density $n=1$ (higher lobes with integer $n>1$ are not shown), and which is surrounded by the SF phase.

There are two qualitatively different ways to make a transition from the MI to the SF ${ }^{1}$ The generic MI-SF transition is driven by addition or subtraction of small numbers of particles to the incompressible MI phase, the total energy cost for which is given by the distance in $\mu$-direction from the nearest phase boundary. Since additional particles or holes (which Bose condense at $T=0$ ) can move freely, the gain in kinetic energy can outweigh the interaction energy, leading to the MI-SF transition. Across the generic transition, which is mean-field like in character, the density varies continuously and the single-particle gap closes linearly as a function of the distance from the phase boundary, $E_{\mathrm{g}} \propto \delta$, where $\delta=t-t_{\mathrm{c}}$ or $\mu-\mu_{\mathrm{c}}$ is the distance from the phase boundary. 1

There also exists a MI-SF transition at fixed density, driven by the onset of boson hopping due to the increase of the ratio $t / U$, i.e. by quantum fluctuations. It has been shown that this transition occurs at the tip of the Mott lobe, and that it has a different universality class than the generic transition! In $d$ dimensions, the universality class is that of the $(d+1)$ dimensional $X Y$ model, so that in 1D there is a KosterlitzThouless phase transition at the multicritical point. For this case, the Mott gap $E_{\mathrm{g}} \propto \exp \left(-\mathrm{const} / \sqrt{t_{\mathrm{c}}-t}\right)$ closes exponentially (i.e., very slowly) as a function of the distance from the lobe tip, $\frac{13}{13}$ and strong deviations from the parabolic lobes predicted by mean-field theory 11 are observed in both strongcoupling $13 \mid 3154$ and DMRG results ${ }^{55}$ Another remarkable aspect of the 1D case is the occurrence of multiple MI-SF transitions along lines of constant chemical potential over an extended range of $\mu \lesssim 0.2$ [see Fig. 11 (a)].14|55

The polariton model also shows a series of Mott lobes, in which the polariton density $n_{\mathrm{p}}$ is pinned to an integer (see Fig. 1(b) for the phase boundaries of the $n_{\mathrm{p}}=1$ lobe obtained by DMRG ${ }^{39}$ ). Even for pinned $n_{\mathrm{p}}$ the photon and exciton densities can fluctuate. Deep in the Mott phase and for $n_{\mathrm{p}} \geq 1$,
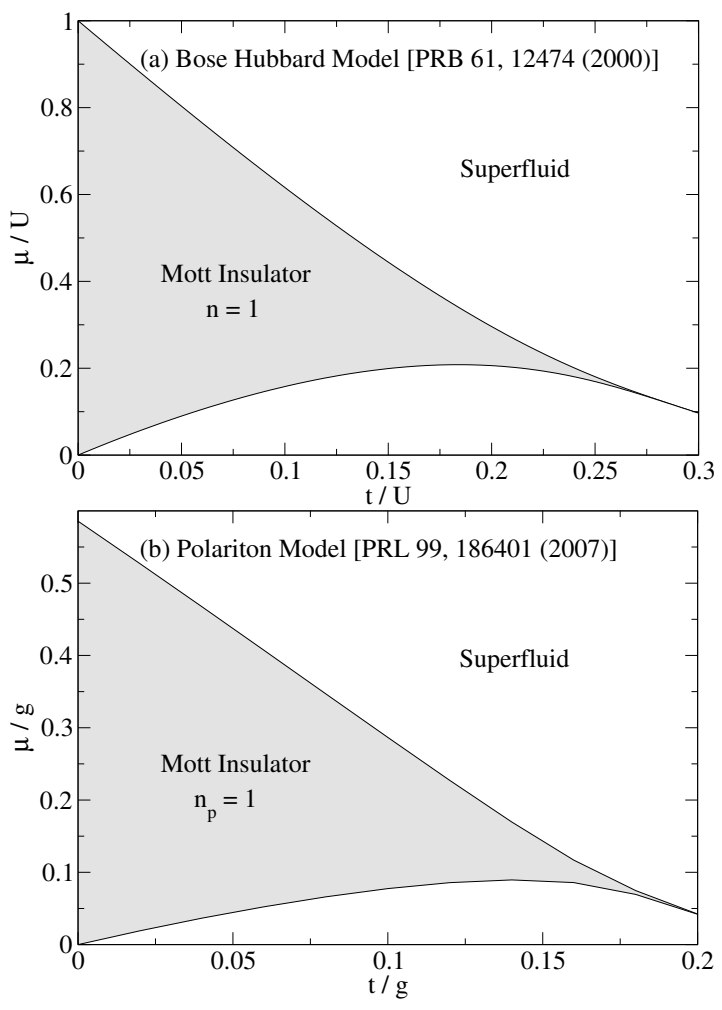

FIG. 1: Zero-temperature phase diagram for (a) the Bose-Hubbard model and (b) the polariton model in 1D. We only show the Mott lobes with density one. These DMRG results were obtained by (a) Kühner et al. ${ }^{[14}$ and (b) Rossini et al. ${ }^{[39}$

we can approximate the ground state by a product over single sites, each of which is described by the Jaynes-Cummings eigenstates (see, e.g., [25])

$$
\begin{aligned}
& \left|n_{\mathrm{p}},-\right\rangle=\cos \theta\left(n_{\mathrm{p}}\right)\left|n_{\mathrm{p}}, \downarrow\right\rangle-\sin \theta\left(n_{\mathrm{p}}\right)\left|n_{\mathrm{p}}-1, \uparrow\right\rangle, \\
& \left|n_{\mathrm{p}},+\right\rangle=\sin \theta\left(n_{\mathrm{p}}\right)\left|n_{\mathrm{p}}, \downarrow\right\rangle+\cos \theta\left(n_{\mathrm{p}}\right)\left|n_{\mathrm{p}}-1, \uparrow\right\rangle,
\end{aligned}
$$

where $\tan \theta\left(n_{\mathrm{p}}\right)=2 g \sqrt{n_{\mathrm{p}}} /\left[2 \chi\left(n_{\mathrm{p}}\right)-\Delta\right], \quad \chi\left(n_{\mathrm{p}}\right)=$ $\sqrt{g^{2} n_{\mathrm{p}}+\Delta^{2} / 4}$, and with eigenvalues $E^{ \pm}\left(n_{\mathrm{p}}\right)=-(\mu-$ $\left.\omega_{0}\right) n_{\mathrm{p}}+\Delta / 2 \pm \chi\left(n_{\mathrm{p}}\right)$. Hence for fixed polariton number $n_{\mathrm{p}}$, the ground state $\left|n_{\mathrm{p}},-\right\rangle$ is a coherent superposition of two states which differ by the state of the atom (or spin) as well as the number of photons; this hybridization provides the connection to exciton polaritons.

The extent of the lobes in both the $\mu$ and $t$ directions diminishes quickly with increasing $n_{\mathrm{p}}$ due to the reduced polaritonpolariton repulsion $U_{\text {eff }}\left(n_{\mathrm{p}}\right)$; the $t=0$ vertical width of the lobes in the Bose-Hubbard model is always $U$. At large values $\zeta t>\omega-\mu$ ( $\zeta$ being the coordination number), beyond those considered in the present work, the polariton model shows an instability! 10

In this work we restrict our discussion to the region in the phase diagram in or close to the Mott lobes with density $n_{\mathrm{p}}=1$ or $n=1$. This lobe is the largest in the polariton model with zero detuning, and quantum effects are most pronounced. A density of one is also the most interesting case for experimental realizations! ${ }^{7 / 24}$ 
All the discussion so far has been for $T=0$. Both experiments and our simulations are carried out at low but finite temperatures, with several important consequences. Strictly speaking, there is no true MI at $T>0$ due to thermal excitations. However, there exist quasi-MI regions which have finite but very small compressibility (see also the discussion of temperature effects later). As long as the density remains close to an integer, these regions may be regarded as Mott insulating. Corresponding "phase diagrams" at finite $T$ have been obtained for both the polariton and the Bose-Hubbard model. 222456 Except for our analysis of temperature effects in Sec. IV] the simulations have been carried out at values of $\beta=3 L$, large enough to ensure that we have an (almost) integer density in the Mott phase.

The Bose-Hubbard model in more than one dimension (and most likely the polariton model as well) exhibits a phase transition from a SF to a normal state (gapless with no phase coherence), related to the well-known $\lambda$ transition in liquid helium, at a temperature $T_{\lambda}{ }^{[2021]}$ This gives rise to an intervening normal region in the phase diagram, between the MI (at small $t / U$ ) and the SF (at large $t / U) \stackrel{56}{\text { In }}$ In $1 \mathrm{D}$ case considered here we have $T_{\lambda}=0$, so that for any $T \neq 0$ only quasi-MI and normal states exist in the thermodynamic limit. However, when the temperature is so low that the SF correlation length in the thermodynamic limit far exceeds the system size $L$, results will be representative of the SF state. Making use of finite size and finite temperature effects, a scaling analysis in fact yields accurate results for the $T=0$ phase boundaries ${ }^{[5357}$ Remarkably, interacting $1 \mathrm{D}$ bosons can be realized using cold atomic gases (the Tonks-Girardeau gas) $\sqrt{58159}$ and are described by the Bose-Hubbard model at low but finite temperatures .32

Similar to Bose fluids, the low-energy excitations in the SF phase are phonons. Within Bogoliubov theory, ${ }^{60}$ these quasiparticles are described by a creation operator $\psi_{k}^{\dagger}=$ $\mathrm{u}_{k} b_{k}^{\dagger}+\mathrm{v}_{k} b_{-k}$, and they have been observed experimentally in ultracold atom systems. ${ }^{61}$ As some of our results can be understood in terms of Bogoliubov theory, let us state some key results for the Bose-Hubbard model. The coefficients of the coherent superpositions of particle and hole excitations are given by 62

$$
\begin{aligned}
& \left|\mathbf{u}_{k}\right|^{2}=\frac{K(k)+n_{0} U+\omega_{k}}{2 \omega_{k}} \\
& \left|\mathbf{v}_{k}\right|^{2}=\frac{K(k)+n_{0} U-\omega_{k}}{2 \omega_{k}}=\left|\mathbf{u}_{k}\right|^{2}-1,
\end{aligned}
$$

with excitation energy

$$
\begin{aligned}
\omega_{k} & =\sqrt{K(k)\left(2 n_{0} U+K(k)\right)}, \\
K(k) & =4 t \sin ^{2}(k / 2) .
\end{aligned}
$$

Here $n_{0}$ is the condensate fraction, equal to $n_{0}=(\mu+t) / U$ in the simple Bogoliubov approach at $T=00^{[23}$ For small $k \approx 0$, we have a linear dispersion $\omega_{k} \approx \pm \sqrt{2 n_{0} t U} k$, and both $\left|\mathrm{u}_{k}\right|$ and $\left|\mathrm{v}_{k}\right|$ are nonzero. For large $k \approx \pi$, the energy dispersion is $\pm\left(-c k^{2}+2 \sqrt{4 t^{2}+2 n_{0} U t}\right)$ and thus free particle like. If we assume $t \gg U$, which is the parameter region where Bogoliubov theory is valid, then $\left|\mathrm{u}_{k}\right|^{2} \approx 1$ and $\left|\mathrm{v}_{k}\right|^{2} \approx 0$ for $k \gg 0$,
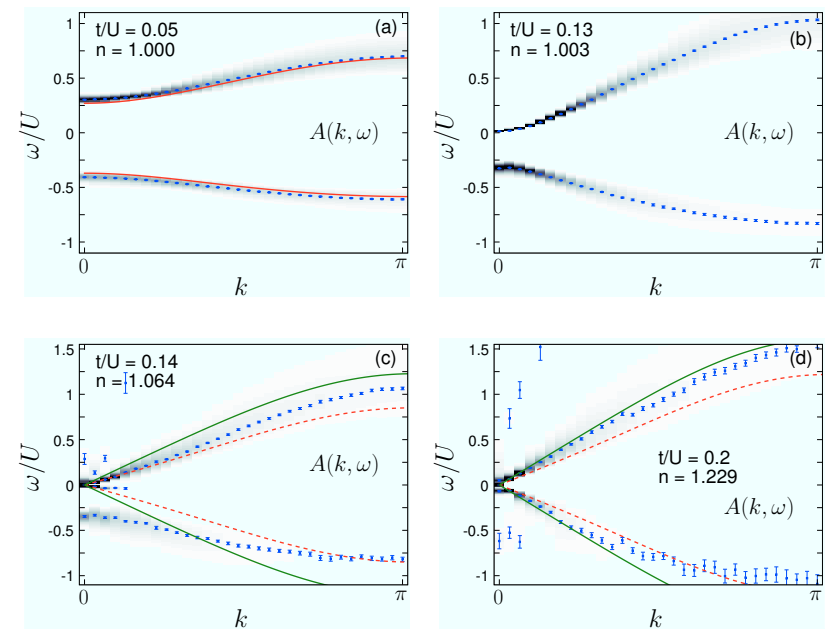

FIG. 2: (color online) Single-boson spectral function $A(k, \omega)$ of the 1D Bose-Hubbard model, for different hoppings $t$ (and total density $n$ ), corresponding to (a) the MI phase, (b) just below the MI-SF transition, (c) just above the transition, and (d) the SF phase. Here $\mu / U=0.5, L=64$ and $\beta U=3 L$. Here and in subsequent spectra, the symbols and errorbars indicate the maxima of the peaks and the associated errors obtained by the maximum entropy method. As discussed in Sec.IV A 2, features with very small spectral weight are difficult to determine accurately. The solid red lines in (a) are mean field results ${ }^{63}$ The solid lines in (c) and (d) are the Bogoliubov results, while the dashed lines are a fourth order approximation (see text) ${ }^{62}$

i.e. only one excitation branch is populated at large momenta. This also holds true for the parameters studied numerically in this work. We further compare to the higher order approximation proposed in Ref. 62. For the Bose-Hubbard model, the latter yields the same equations for $\left|\mathbf{u}_{k}\right|^{2},\left|v_{k}\right|^{2}$ and $\omega_{k}$, but $n_{0}$ is determined self-consistently, allowing for depletion effects.

In the case of free bosons at $T=0$, all particles condense in the same $k=0$ state. However, finite temperature and/or interactions cause a certain fraction of these particles to occupy states of higher energy. Indeed, both for $U \rightarrow 0$ (noninteracting bosons) and $n_{0} \rightarrow 0$ (high temperature limit) we have $\left|\mathrm{u}_{k}\right|^{2}=1,\left|\mathrm{v}_{k}\right|^{2}=0$. Moreover, with decreasing $U$ or $n_{0}$, $\left|\mathrm{v}_{k}\right|^{2}$ approaches zero most quickly at large $k$ since in this case $K(k) \gg n_{0} U$ so that $\omega_{k} \approx K(k)$, canceling the term $-\omega_{k}$ in the expression for $\left|v_{k}\right|^{2}$. This will explain the temperature evolution of the single-particle spectrum shown in Sec. IV

\section{A. Bose-Hubbard model}

Despite the extensive literature on this model, there are few nonperturbative results available for the spectra, as mentioned in Sec. I. Therefore, we investigate the single-boson spectral function $A(k, \omega)$ and the dynamic structure factor $S(k, \omega)$, with results shown in Figs. 2 and 4 . 


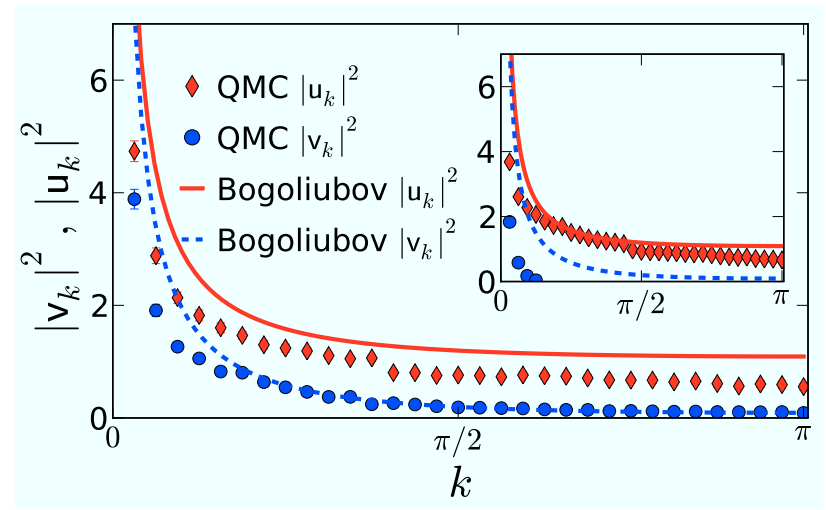

FIG. 3: (color online) Quasiparticle weights $\mathrm{u}_{k}$ and $\mathrm{v}_{k}$ of the gapless modes at $t / U=0.2$. The symbols are integrated intensities from QMC and maximum entropy, the lines are the predictions from Bogoliubov-theory. The inset shows data at $t / U=0.14$. Again, $\mu=0.5, L=64$ and $\beta=3 L$.

\section{Single-particle spectrum}

Menotti and Trivedi reviewed previous work on the singleparticle spectrum, and presented results from a random phase approximation $\sqrt{23}$ Their main findings are as follows. For large $t / U$, a weakly interacting SF exists, and the spectrum consists of the usual two gapless phonon modes which exhaust the sum rule for $A(k, \omega)$. Reducing $t / U$, two additional gapped modes appear at small $k$ whose spectral weight increases upon approaching the quantum phase transition. At the transition, one of the phonon modes evolves into the particle or hole mode (depending on which of the gaps $E_{\mathrm{g}, \mathrm{p}}, E_{\mathrm{g}, \mathrm{h}}$ is smaller), whereas one of the gapped modes in the SF becomes a gapped mode in the MI. Menotti and Trived ${ }^{23}$ argued that the appearance of gapped modes and the redistribution of spectral weight from coherent phonon modes to incoherent gapped modes indicate the strongly correlated nature of the SF state near the transition. Let us point out that particle and hole dispersions in the MI have been calculated by several

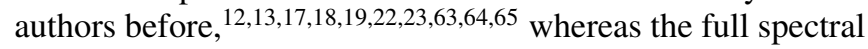
function of the MI (which also reveals the spectral weight and the width of the excitations) was only shown in [18|23].

Our numerical results for the single-particle spectral function $A(k, \omega)$ are shown in Fig. 2. The four different values of the ratio $t / U$ cover the range in which the generic MI-SF transition takes place. According to Fig. 11.a), for the chosen value of $\mu / U=0.5$ the transition occurs at $t / U \approx 0.14$. In each panel we also report the total density $n$ to three decimal places, although our simulations provide much higher accuracy. The MI [(a) and (b)] exhibits the familiar gapped particle and hole bands. ${ }^{13}$ The additional particles exhibit a free-particle dispersion since the energy penalty for double occupation is the same at every site. In particular, we see in Fig. 2(a), (b) that the particle band width is $8 t$ (the factor of two arising from the fact that particle hopping involves a doubly occupied site), whereas the hole bandwidth is $4 t$. The Mott gap decreases with increasing $t$ and a symmetry of particle and hole bands emerges ${ }^{1 / 20 \mid 24}$ In addition to our QMC results we plot the mean-field dispersion ${ }^{63}$ in Fig. 22a). For larger $t / U=0.13$, mean-field theory already predicts a superfluid, although the critical hopping in $1 \mathrm{D}$ is $t_{\mathrm{c}} / U \approx 0.14$.

In the SF phase [Fig. 2(c),(d)], we obtain the expected Goldstone modes with linear dispersion at small $k$. Additionally, we see two gapped signals which we relate to the gapped modes discussed by other authors. ${ }^{[1 / 1923}$ Whereas the negative-energy gapped mode is clearly visible in Fig. 2(c) just above $t_{\mathrm{c}}$, the gapped modes have almost disappeared in Fig. 2(d). Since we approach the phase transition above the lobe tip $(\mu / U=0.5)$ the particle band becomes the gapless mode and carries more spectral weight, while the gapped hole band evolves into a gapped mode in the SF. This agrees well with the findings of Menotti and Trivedi ${ }^{23}$ In accordance with Bogoliubov theory, the excitations in the SF phase are freeparticle like for large $k$. The bandwidths of the excitations both in the MI and the SF phase scale roughly linearly with $t$.

In Figs. 2(c) and (d) we also show results for the phonon dispersion $\pm \omega_{k}$ (without taking into account the weights $\left|\mathrm{u}_{k}\right|$, $\left.\left|v_{k}\right|\right)$ from Bogoliubov theory as well as the higher-order approximation of Ref. 62. Whereas the simple Bogoliubov approach (neglecting depletion of the condensate) agrees quite well with our data despite the rather small value of $t / U$, we do not find the higher order approach to be systematically better. In particular, at large $k$, the phonon bandwidth is noticeably underestimated, which may be a result of an overestimate of depletion effects (these are most visible at large $k$ ). The agreement with Bogoliubov theory at small $k$ coincides with the findings of Menotti and Trivedi ${ }^{23}$ Rey et al ${ }^{62}$ found the higher order approximation to be consistent with numerical results for other observables but do not show the spectra seen in Fig. 2. Note that these authors consider larger particle densities $n \geq 5$ where the Bogoliubov-type approximations are more reliable. Finally, we tried to use our QMC results for the superfluid fraction for $n_{0}$ in the expressions obtained from Bogoliubov theory, but the results are worse than for $n_{0}=n$.

The spectral weight of the excitations decreases with increasing $k$ in all spectra of Fig. 2, although this is more pronounced in the SF phase than in the MI. In Fig. 3 we show the quasiparticle weights of the massless modes in the SF phase, obtained by integrating over the quasiparticles peaks in the spectra, and compare them to Bogoliubov theory (Eqs. 9 and 10 . We verified that the QMC spectra satisfy the sum rule. The spectral weight of the lower branch decreases more quickly, consistent with the Bogoliubov picture. However, Bogoliubov theory overestimates the quasiparticle weights, especially at small $k$. Besides, there is a significant broadening of the peaks on approaching the zone boundary. At strong coupling close to the phase transition (inset of Fig. 3), the quasiparticle weight of the lower branch decays much more quickly than Bogoliubov theory would predict.

Sound velocity. The sound velocity $v_{\mathrm{s}}=\left.\frac{\partial \omega_{k}}{\partial k}\right|_{k \rightarrow 0}$ of the phonon excitations in the SF phase was calculated for the Bose-Hubbard model by Menotti and Trivedi using a random phase approximation. ${ }^{[2]}$ They concluded that $v_{\mathrm{s}}$ vanishes at the generic transition, but remains nonzero when crossing the multicritical point .1923 In their results, there is a very sharp downturn of $v_{\mathrm{s}}$ toward zero close to $t_{\mathrm{c}}$. We are not aware of 

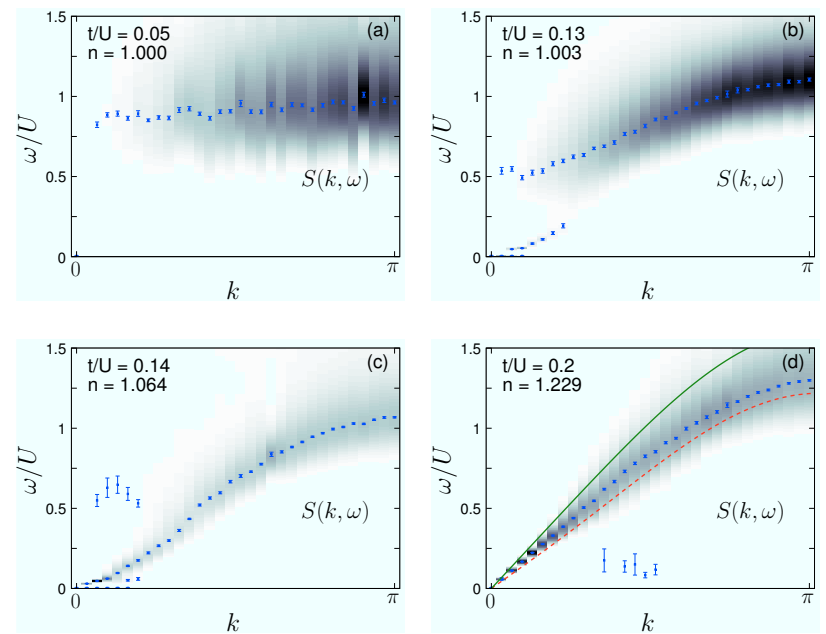

FIG. 4: (color online) Dynamic structure factor $S(k, \omega)$ of the BoseHubbard model for the same parameters as in Fig. 2 Panel (d) includes the same analytical approximations as Fig. 22,d).

any calculations of $v_{\mathrm{s}}$ for the polariton model.

From our QMC simulations, we can determine $v_{\mathrm{s}}$ from linear fits to the spectrum. Apart from the limited accuracy of the maximum entropy inversion, this works quite well away from $t_{\mathrm{c}}$. In agreement with Bogoliubov theory, we find for the Bose-Hubbard model a linear dependence $v_{\mathrm{s}} \propto\left|t-t_{\mathrm{c}}\right|$ and good agreement of results for $L=32$ and 64 .

Determining the behavior of $v_{\mathrm{s}}$ as $t \rightarrow t_{\mathrm{c}}$ is more difficult for two reasons. First, the phonon spectrum becomes nonlinear due to finite-temperature effects (see discussion below), rendering linear fits ill-defined. Second, the position of the phase transition changes with system size, so that no reliable finite-size scaling of $v_{\mathrm{s}}$ can be carried out. The situation is similar for the polariton model, and we therefore do not show results for $v_{\mathrm{s}}$ here, leaving this as an interesting issue for future work.

\section{Dynamic structure factor}

The single-particle spectral function provides information about the energy and lifetime of particles or holes added to the interacting ground state. In contrast, the dynamic structure factor-corresponding to the imaginary part of the dressed particle-hole propagator-yields insight into the density fluctuations in the ground state. In general the two quantities do not exhibit the same features. However, for broken U(1) gauge symmetry in the SF phase, they are both dominated by the same single-particle excitations (phonons). ${ }^{26}$ We find this statement to hold in 1D even though no symmetry breaking occurs.

The density operator in the Bose-Hubbard model is $\rho_{k}^{\dagger}=$ $\sum_{l} e^{-i k l} \hat{n}_{l}$. For $k=0$, we have $\rho_{k}^{\dagger}=\sum_{l} \hat{n}_{l}$, and $S(k, \omega)$ has a trivial contribution at $\omega=0$ which we dismiss by considering $\widetilde{\rho}_{k}^{\dagger}=\sum_{l} e^{-i k l}\left(\hat{n}_{l}-\left\langle\hat{n}_{l}\right\rangle\right)$. The above-mentioned relation to particle-hole excitations becomes evident by rewriting the density operator as $\rho_{k}^{\dagger}=\sum_{q} b_{q+k}^{\dagger} b_{q}$.

We show results for $S(k, \omega)$ in Fig. 4 According to Huber et al. ${ }^{19} S(k, \omega)$ in the MI phase should exhibit a continuum of particle-hole excitations, starting at $\omega=E_{\mathrm{g}}$ due to the Mott gap in the single-particle spectrum (see Fig. 2). For the parameters in Fig. 4(a), $E_{\mathrm{g}} / U \approx 0.7$. The dispersion of the particle and hole bands is very weak, Note that we find no agreement with the two single-particle excitations $E_{\mathrm{g}}^{\mathrm{p}}+\epsilon_{\mathrm{h}}(k)$, $E_{\mathrm{g}}^{\mathrm{h}}+\epsilon_{\mathrm{p}}(k)$ discussed by Huber et al. This may be a result of their mean-field treatment of the two-dimensional case. Our results do agree qualitatively with exact numerical results on small clusters. ${ }^{15}$

For larger $t / U$, the Mott state contains nontrivial density fluctuations, and the upper band in $S(k, \omega)$ acquires some $k$ dependence. The energy of the excitations in $S(k, \omega)$ [following $\left.\sum_{q}\left\{\epsilon_{\mathrm{h}}(q)+\epsilon_{\mathrm{p}}(k-q)\right\}\right]^{19}$ generally increases with increasing $k$. This is obvious from the momentum dependence of the particle and hole bands in $A(k, \omega)$, and also agrees with the expectation that long-wavelength density fluctuations in a Mott state require less energy than fluctuations with short periods in real space.

For $t \lesssim t_{\mathrm{c}}$ in Fig. 4(b), we find a low-energy mode with nonlinear dispersion, which we interpret as a precursor of the linear excitations of the SF phase [see panel (d)]. Even for $t \gtrsim t_{\mathrm{c}}$ [Fig. 4(c)], the gapless low-energy mode in our numerical results is not linear. A linear spectrum is a result of the condensation of bosons in the SF phase, but is not expected in the normal phase. Since our simulations are done at finite temperature, and because the phase coherence length is small close to $t_{\mathrm{c}}$, we can understand the absence of a clear, linear signature in Fig. 4(c). Going to larger $t_{\mathrm{c}}$, we indeed see linear excitations near $k=0$ [Fig. 4(d)]. Similar effects are expected for the single-particle excitations, but are difficult to see on the scale of Fig. 2 Coming back to Fig. 4(c), away from $k=0$, we find a free-particle like contribution, similar to the case of the MI. This excitation carries negligible spectral weight near $k=0$.

Apart from finite-temperature effects, these features are qualitatively similar to the excitations discussed by Huber et al., 19 namely a gapless sound mode (related to phase and density modulations) dominant at small $k$, and a massive mode (corresponding to exchange between condensate and noncondensate at fixed density) acquiring spectral weight at $k>0$. Additionally, we see in Fig. 4/c) the (weak) signature of a gapped mode at small $k$, the nature of which we cannot determine from our present simulations. For $t / U=0.2$ [Fig. 4(d)] the excitation "band" in $S(k, \omega)$ follows closely the Bogoliubov mode, in accordance with the discussion at the beginning of this section.

At this point, a comment concerning the accuracy of the spectra obtained from the maximum entropy inversion is in order. The spectral weight of the features visible in density plots such as Fig. 4[d) varies over orders of magnitude. Some very weak signals, such as the group of points located at around $k=\pi / 2$ below the main excitation band (with a weight that is a factor 10000 smaller than that of the dominant features), are expected to be artifacts. We shall see below that in the polariton model, there actually exist real excitations with very 

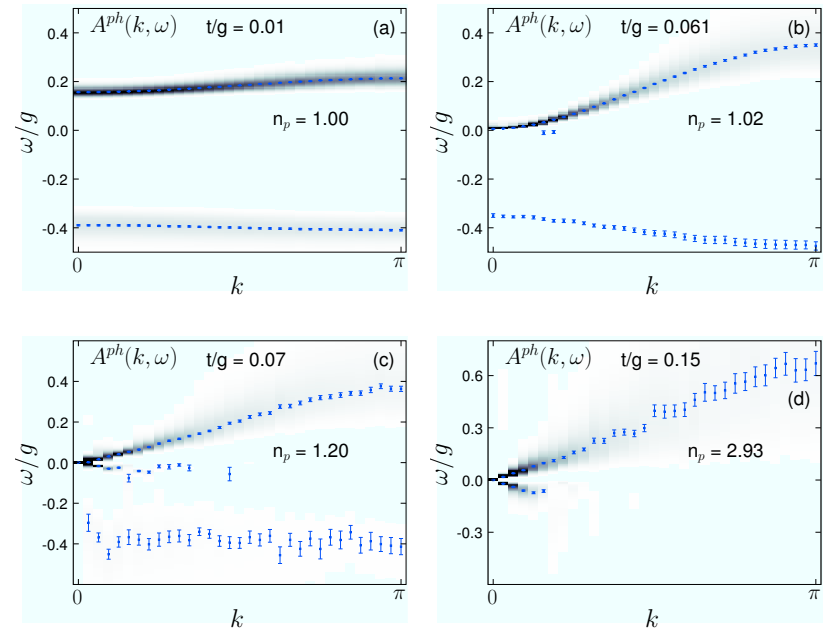

FIG. 5: Single-photon spectral function $A^{\mathrm{ph}}(k, \omega)$ of the 1D polariton model at $\mu / g=0.4$ for different hoppings $t$, corresponding to (a) deep in the MI, (b) just below the MI-SF transition, (c) just above the transition, and (d) in the SF phase. Here $\beta g=3 L$ and $L=64$. With increasing $t$, the density plots are more and more "overexposed" to see less dominant features.

small spectral weight which are easy to miss in the maximum entropy inversion. To reliably study such excitations, analytical approaches (if available) are clearly superior. ${ }^{25}$

Our findings for the dynamic structure factor are consistent with previous numerical results on small systems $(L=$ $10,20) ! \frac{1516}{\sqrt{16}}$ We can confirm the broadening of the excitations with increasing $k$ in the SF phase, $\frac{16}{16}$ related to two-particle continua ${ }^{\sqrt{19}}$ However, the maximum entropy method is not capable of resolving fine structures as (generically) seen in exact diagonalization results for small clusters ${ }^{15}$

\section{B. Polariton model}

For the polariton model, the only published results on dynamic properties are for the single-particle spectrum of the MI phase at zero temperature. ${ }^{2425}$ As pointed out before, the nature of the conserved particles in the polariton model is determined by the detuning. We start by discussing the case $\Delta=0$ for which the polaritonic character of the excitations is most pronounced. This can readily be seen from Eq. (8), where $\left|n_{\mathrm{p}}, \downarrow\right\rangle$ and $\left|n_{\mathrm{p}}-1, \uparrow\right\rangle$ contribute with equal weight.

\section{Single-particle spectrum}

In Fig. 5] we show our QMC results for the single-photon spectral function. As for the Bose-Hubbard model, the values of the ratio $t / g$ range from deep in the Mott phase across the generic transition well into the SF phase. According to a finite size scaling analysis for $\mu / g=0.4$, the phase transition occurs at $t_{\mathrm{c}} / g=0.0626(1)$ (see Fig. 14), in agreement with Fig. 1 b). Hence panels (a) and (b) are for the MI regime,
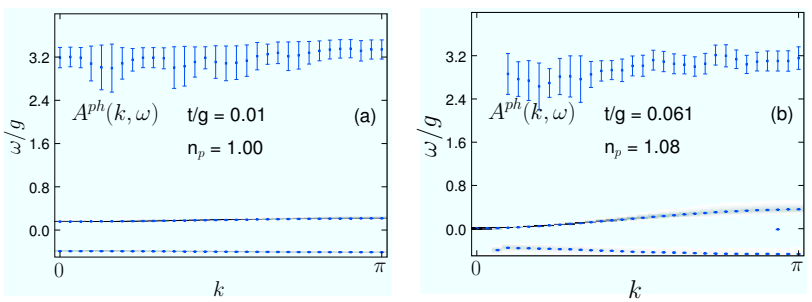

FIG. 6: Single-photon spectral function in the Mott phase for the same parameters as in Fig.5. showing additional excitations at higher energies.

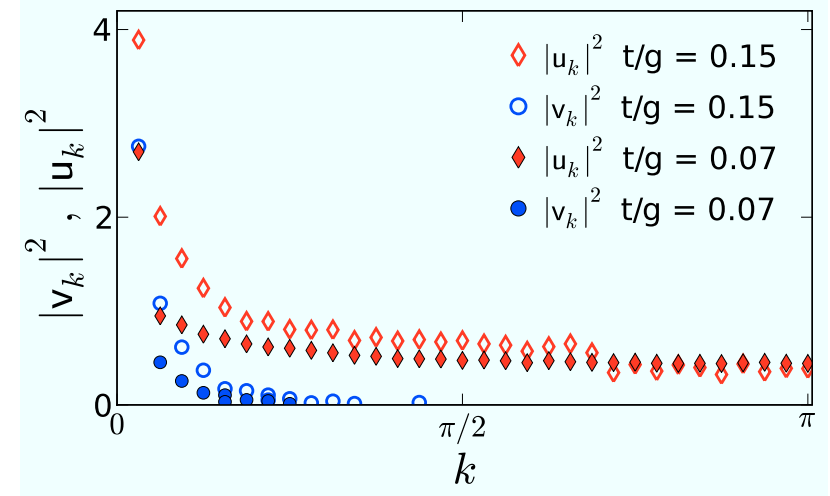

FIG. 7: (color online) Quasiparticle weights $\mathrm{u}_{k}$ and $\mathrm{v}_{k}$ of the gapless modes of the polariton model, similar to Fig. 3

whereas (c) and (d) are for the SF phase.

The results in the MI shown in Fig. 5(a), (b) agree well with previous numerical work ${ }^{24}$ Similar to the Bose-Hubbard model, there exist particle and hole bands, separated by the Mott gap. It is important to stress that although we add bare photons to the system, the particle and hole excitations reflect the properties of the polaritons in the system. Whereas the ratio of particle and hole bandwidths is two to one in the Bose-Hubbard model, it depends on the character of the quasiparticles (polaritons) in the polariton model and varies with detuning ${ }^{24}$ With increasing $t / g$, the gap closes and the bandwidths of excitations increase (effective masses decrease).

Recent analytical work revealed the existence of so-called upper polariton modes at higher energies, which represent an important difference between the Bose-Hubbard model and the polariton model ${ }^{[25]}$ For the Mott lobe with $n_{\mathrm{p}}=1$, only one such (particle) band exists, corresponding (for small enough $t / g)$ to a transition between the ground state $\left|n_{\mathrm{p}}=1,-\right\rangle$ and the state $\left|n_{\mathrm{p}}=2,+\right\rangle$ (see Eq. (8)). The weight of this highenergy excitation is very small compared to the dominant particle and hole modes discussed above ( 0.04 as compared to 1.46 for the $k=0$ atomic-limit results in [25]); with increasing $t / g$ the weight difference becomes even larger ${ }^{[25}$ The energy splitting between the - and + branches of eigenstates increases further for detuning $\Delta \neq 0$ (Fig. 2 in [7]).

The upper polariton mode is not visible in Figs. 5 (a) or (b). Excitations with small spectral weight are notoriously difficult to see using QMC in combination with maximum entropy. In 

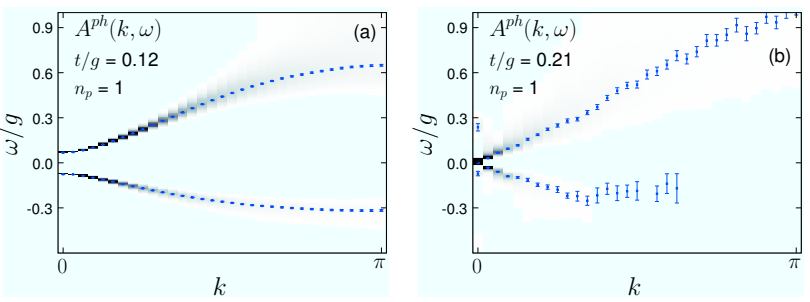

FIG. 8: Single-photon spectrum $A^{\mathrm{ph}}(k, \omega)$ of the polariton model along the line $n_{\mathrm{p}}=1$ crossing the Kosterlitz-Thouless transition. Here $L=64$ and $\beta g=3 L$.

the present case, this is aggravated by the fact that the resolution of maximum entropy decreases at high energy. Nevertheless, we see a signature of the upper polariton band in Fig. 6, and the latter is also present (but not shown) in the high-temperature data of Fig. 12.a); high-energy features are easier to resolve in QMC/maximum entropy at higher temperatures. From the eigenvalues of the states (8) we can determine the excitation energy of the upper mode in the atomic limit as $w_{p}^{+} / g=-\left(\mu-\omega_{0}\right) / g+(\sqrt{2}+1) \approx 3$ for the parameters of Fig. 6, in reasonable agreement with our results in Fig. 6. a) given the ill-conditioned nature of the problem under consideration. Note that the upper polariton mode can be seen even close to the phase transition in Fig. 6 b). The weight of the upper mode in Fig. 6 is about a factor of 100 smaller than that of the conventional particle and hole excitations. Although the upper polariton mode exists also in other results for the single-particle spectrum in the Mott phase (Figs. 8, 12 and 13, we focus on the low-energy conventional modes with large spectral weight. The latter can be determined accurately from our simulations, and will be the dominant feature in experiments.

Figures $5(\mathrm{c})$ and (d) contain the first spectra of the polariton model in the SF phase. There is a clear signature of the gapless phonon modes starting at $k=\omega=0$, with linear dispersion at small $k$. In the SF phase but close to the transition, we see an additional gapped mode at $\omega<0$ [Fig. 5.c)]. Our results at these and at further couplings $t / g$ (and $t / U$ ) suggest that these gapped modes disappear more quickly with increasing $t / g$ than for the Bose-Hubbard model, which can be explained in terms of the photonic SF expected for the present parameters (see below) ${ }^{[29}$ Note that a simple Bogoliubov type theory for the polariton model does not exist, due to the composite nature of polariton excitations.

Figure 7 shows the quasiparticle weights. The general shapes resemble the Bose-Hubbard model case (Fig. 3), but the lower branch decays very quickly in the polariton model even at $t / g=0.15$ quite far from the phase transition. This may be attributed to the fact that the energy cost for particle and hole excitations is different due to the dependence of $U_{\text {eff }}$ on $n_{\mathrm{p}}$. Again, most of the spectral weight in the SF phase is found at small $k$.

Fixed density. In Fig. 8 we show the single-photon spectrum across the fixed density transition $\left(n_{\mathrm{p}}=1\right)$, obtained by selecting configurations only at that density. In Ref. 40 ,

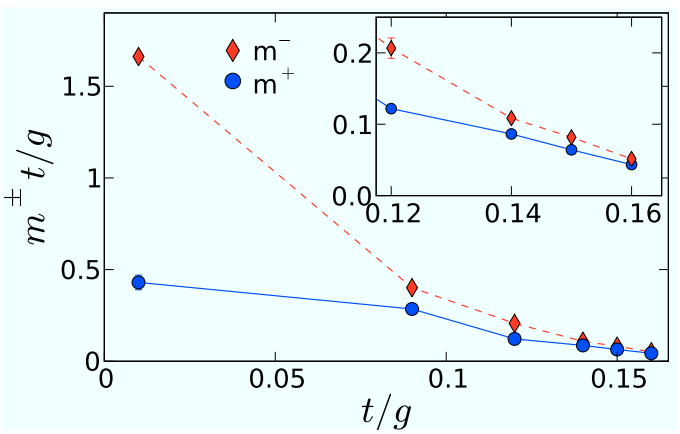

FIG. 9: (color online) Effective particle $\left(\mathrm{m}^{+}\right)$and hole masses $\left(\mathrm{m}^{-}\right)$ along the line $n_{\mathrm{p}}=1$, as obtained from fits to the bands in $A^{\mathrm{ph}}(k, \omega)$ near $k=0$.
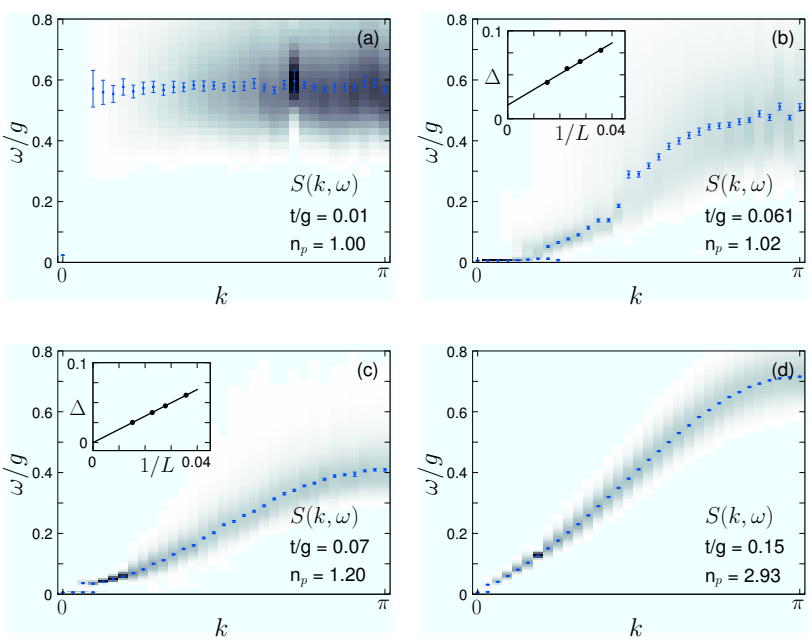

FIG. 10: Polariton dynamic structure factor $S(k, \omega)$ for the same parameters as in Fig. 5] The insets show an extrapolation of the Mott gap at small $k$ to $L \rightarrow \infty$.

the critical hopping was determined as $t_{\mathrm{c}} / g=0.198$ [cf Fig. 11.b)]. The spectra in both the MI and the SF look very similar to those across the generic transition shown above. This may be different very close to the multicritical point, but this regime is most demanding numerically if the results are to be used in a maximum entropy inversion.

From the spectra obtained at constant density in the MI, we can estimate the effective particle and hole masses by fitting a quadratic dispersion to the bands in the vicinity of $k=0$. In the Bose-Hubbard model, there is an emergent particle-hole symmetry on approaching the lobe tip, $\stackrel{1120}{ }$ and similar behavior is suggested by the evolution of the particle and hole bands with increasing $t / g$ also in the polariton model. For fixed polariton density, the two masses approach each other and vanish at the phase transition. This has been demonstrated in 2D based on a strong-coupling approach ${ }^{25}$ In the region not too close to the phase transition, where stable fits can be obtained, Fig. 9 confirms this observation also in 1D. 


\section{Dynamic structure factor}

The evolution of the polariton dynamic structure factor $S(k, \omega)$ across the MI-SF transition is shown in Fig. 10 . Remarkably, the results look very similar to those for the BoseHubbard model. Close to the atomic limit $[t / g=0.01$ in Fig. 10,a)] we see a gapped, almost flat feature with energy $\omega \approx 0.6 \mathrm{~g}$. A look at the corresponding single-particle spectrum in Fig. 8(a) reveals that this value is identical to the Mott gap. The almost flat particle and hole bands cause a very weak dispersion also for the particle-hole excitations visible in $S(k, \omega)$. It is useful to remember that it is the effective polariton-polariton repulsion mediated by the atom-photon coupling that determines the Mott gap. For a single site and $n_{\mathrm{p}}=1$ (i.e., for the case of adding a second polariton),

$$
U_{\text {eff }}(1)=2 \sqrt{g^{2}+(\Delta / 2)^{2}}-\sqrt{2 g^{2}+(\Delta / 2)^{2}}-\Delta / 2 .
$$

For zero detuning $(\Delta=0), U_{\text {eff }}(1) / g=2-\sqrt{2} \approx 0.59$.

As for the Bose-Hubbard model, the excitations in $S(k, \omega)$ acquire a noticeable dispersion with increasing $t / g$, and the $k=0$ gap closes. Figures 10 (b) and (c) are both close to the phase transition. An inspection of the $k=0$ region shows a weak linear mode with very small slope $O(0.01)$, corresponding to the small superfluid density existing in both $L=64$ systems. The massive mode extends to $k=0$ with a tiny intensity (thus not visible in the figure). An extrapolation of the gap to $L=\infty$ (insets) shows that it scales to zero in Fig.10.c), but stays finite at the smaller hopping in Fig. 10(b). Indeed, a finite size scaling of the superfluid density (discussed later) implies that Fig. 10(b) is just below the phase transition. In addition to finite size effects, we again observe finite temperature effects in the form of deviations from the expected linear spectrum close to $t_{\mathrm{c}}$ (see discussion for the Bose-Hubbard model). For even larger $t / g$, the spectrum exhibits a single linear mode at small $k$. Similar to the spectral function, gapped modes seem to be suppressed quickly in the SF phase.

Clearly, the polariton dynamic structure factor $S(k, \omega)$ represents a useful probe to distinguish between the MI and the SF phases. We have argued before that the polariton MI has fluctuations in the photon and exciton density, whereas the polariton density is pinned.

We now demonstrate that the exciton (atom) and photon structure factors, $S^{\text {at }}(k, \omega)$ and $S^{\mathrm{ph}}(k, \omega)$, shown in Fig. 11 . do not reflect this fact, and therefore cannot be used to characterize the nature of the Mott state. To this end, it is important to notice that the Jaynes-Cummings Hamiltonian has two branches of eigenstates $\left|n_{\mathrm{p}},+\right\rangle$ and $\left|n_{\mathrm{p}},-\right\rangle$ (the latter containing the ground state, see also Eq. (8)) with the same polariton number but different energy. ${ }^{37}$ In the atomic limit, the dynamic structure factor for photons and excitons [Eq. (5)] is dominated by the contributions $\left\langle n_{\mathrm{p}},-\left|\hat{\rho}_{k}^{\dagger}\right| n_{\mathrm{p}},-\right\rangle$ (with $\omega=$ 0 ) and $\left\langle n_{\mathrm{p}},+\left|\hat{\rho}_{k}^{\dagger}\right| n_{\mathrm{p}},-\right\rangle$ (with energy $\omega=2 \sqrt{n g^{2}+\Delta^{2} / 4}$, equal to 2 in Fig. 11). Any additional peaks at finite $t / g$ have much smaller spectral weight and cannot be accurately resolved by our method. However, since the matrix elements of exciton and photon density operators for the combination $\left\langle n_{\mathrm{p}},+\left|\hat{\rho}_{k}^{\dagger}\right| n_{\mathrm{p}},-\right\rangle$ have the same modulus but opposite sign,
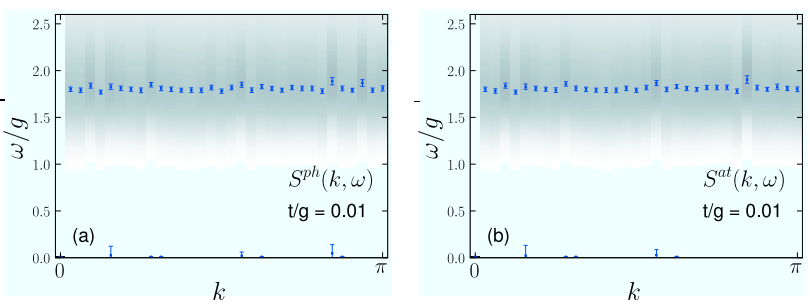

FIG. 11: Dynamic structure factor for excitons $\left(S^{\text {at }}\right)$ and photons $\left(S^{\mathrm{ph}}\right)$ for the same parameters as Fig. 10.a).
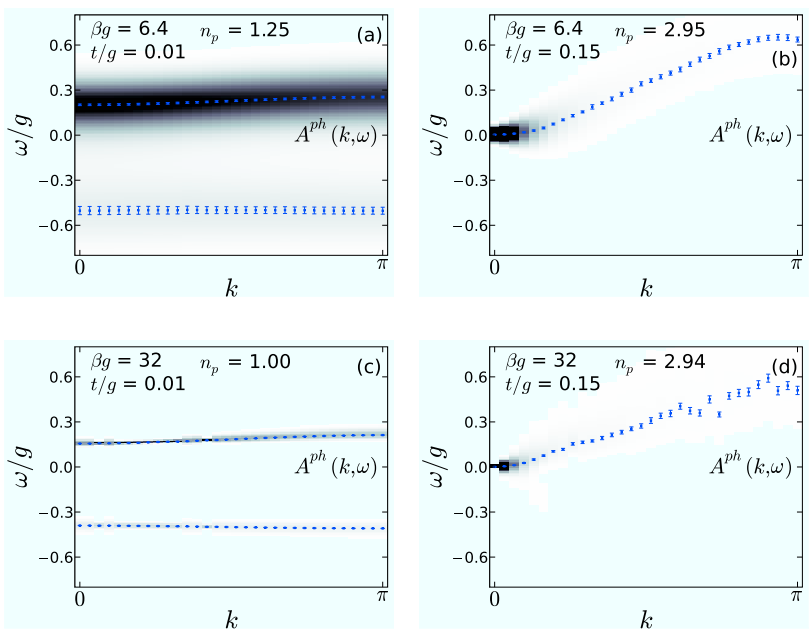

FIG. 12: Temperature dependence of $A^{\mathrm{ph}}(k, \omega)$ in the polariton model at $\mu / g=0.4$, in the MI (left) and in the SF phase (right). Here again $L=64$. Corresponding results at $\beta g=192$ can be found in Figs. 5.a),(d).

the dominant contributions to $S^{\text {at }}(k, \omega)$ and $S^{\mathrm{ph}}(k, \omega)$ cancel in the case of the polariton structure factor $S(k, \omega)$. The dispersionless excitations near $\omega / g=2$ seen in Fig. 11 are therefore absent in $S(k, \omega)$, as confirmed by our data. Hence, while the upper polariton modes in the single-particle spectrum have small but finite weight, here their contribution is zero. Consequently, the polariton dynamic structure factor closely resembles $S(k, \omega)$ of the Bose-Hubbard model.

\section{Temperature effects}

Experimental realizations of Bose-Hubbard models using cold atomic gases are usually prepared very close to zero temperature (nK range). In contrast, due to the strong matter-light coupling achievable in cavities, realizations of polariton models offer a chance of operation at significantly higher temperatures. The critical temperature at which the MI state starts to lose its characteristic integer density has been estimated for the polariton model as $T^{*} / g \approx 0.03 .^{2438}$ For feasible values of the coupling $g, T^{*}$ falls into the $\mathrm{mK}$ range. Generally, Mott-like physics is expected as long as the Mott gap is significantly larger than the thermal energy (and the number 
of particle-hole excitations is small). The finite-temperature physics of the Bose-Hubbard model has been analyzed by several groups. $[5646667]$ Here we consider the effect of low but finite temperatures on the excitation spectra of the polariton model. This also provides information about the sensitivity of the results to the (necessarily finite) value of $\beta$ used in our simulations.

The present method permits calculation of spectra also outside the MI, i.e., in the SF and the normal phase. We have pointed out above that, strictly speaking, there is no SF phase at $T \neq 0$ in 1D. Nevertheless, SF like properties can be seen for $T$ sufficiently small. The results in Fig. 12 underline the discussion of finite-temperature effects on the dispersion in the SF near $k=0$. The particle excitation is obviously not linear in panels (b) and (d), for which the temperature is higher than in Fig. 5 .

Results for $A^{\mathrm{ph}}(k, \omega)$ are shown in Fig. 12 At finite but low temperature [Fig. 12 (c,d)] they still closely resemble the results at $T \approx 0$ in Figs. 5(a) and (d). At high temperature [Fig. 12 (a,b)], we observe strong broadening of the particle band at all $k$, and strongly suppressed spectral weight for hole excitations. Existing work for the Bose-Hubbard model finds that at finite temperature additional multi-particle and hole bands arise ${ }^{66}$ We see an additional excitation for $\beta g=4.4$ and $t / g=0.01$ at an energy of $\omega / g \sim 3.1$. The weight of that excitation is about 50 times smaller than the weight of the main peak with energy $\omega / g \sim 0.2$ and thus not shown in Fig. 12 (a). This excitation is consistent with the upper polariton mode discussed above.

We note that a broadened "gapped" spectrum is compatible with a density that deviates from the integer value characteristic of the MI, and this has to be kept in mind for potential applications relying on integer density. The numerical results for the total density are shown in each of the panels, demonstrating that despite the large particle-hole gap the polariton density deviates significantly from the low-temperature value $n_{\mathrm{p}}=1$ for the parameters of Fig. 12.a).

Some of the features observed in the SF phase can be explained by means of Bogoliubov theory for the Bose-Hubbard model. In particular, we have discussed above that with increasing temperature (where the condensate fraction $n_{0} \rightarrow 0$ ) the spectral weight of the negative energy branch vanishes first at large $k$, in agreement with our numerical results. In addition the broadened positive energy branch no longer has a clear linear behavior at small $k$.

We would like to point out that not only finite temperature but also disorder is an inevitable feature of experimental realizations of coupled cavity systems. Although not studied here directly, it has been stated $\sqrt{38}$ that the effect of disorder (in the form of local variations of the parameters $\omega_{0}, g$ and $t$ ) has similar consequences as finite temperature.

\section{Detuning}

The detuning between the cavity photon mode and the atomic level splitting is an important parameter in the polariton model which is absent in the Bose-Hubbard model. Its
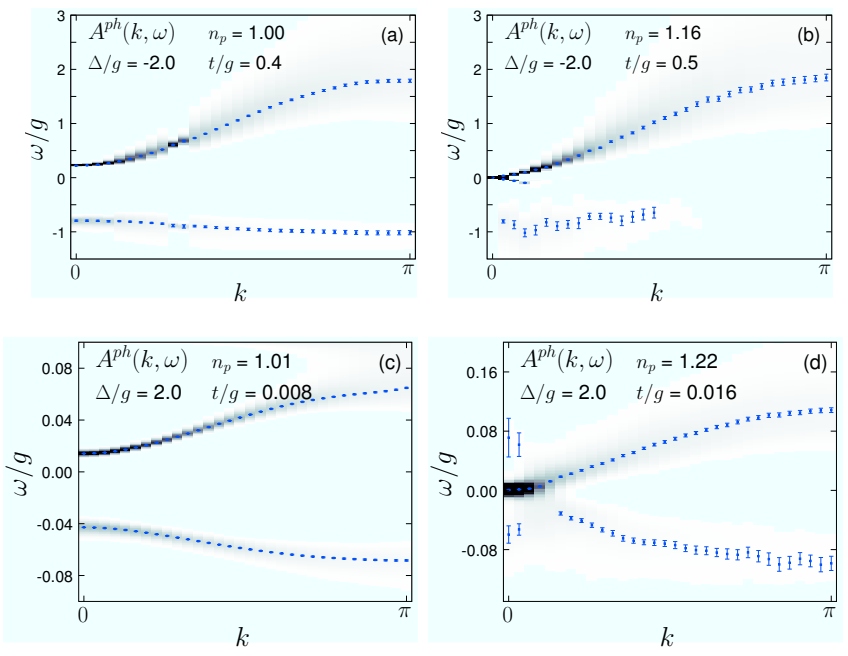

FIG. 13: Single-photon spectra with detuning $\Delta=\epsilon-\omega_{0}$ for the excitonic case $\Delta / g=-2, \mu / g=-0.5$ (a,b) and photonic case $\Delta / g=2, \mu / g=0.64(\mathrm{c}, \mathrm{d})$, in the MI (a,b) and in the SF (c,d). Here $L=64$ and $\beta g=3 L$.

influence on the physics has been discussed before. ${ }^{24|27| 53}$ Detuning can also be easily changed experimentally, motivating a calculation of the excitation spectra for $\Delta \neq 0$. Our results are shown in Fig. 13

The extent of the different phases, namely excitonic or polaritonic MI and photonic or polaritonic SF in the phase diagram has been analyzed for a two-site system. 27 The way to distinguish between the polaritonic SF and the photonic SF is to monitor fluctuations in the exciton occupation number (pinned in the photonic SF but fluctuating in the polaritonic $\mathrm{SF})$. The conclusion has been that for $t \approx|\Delta|$ a polaritonic SF exists only for $\Delta / g<-1$ This would match with the conjectured photonic nature of the SF near the lobe tip in two dimensions $[53$ However, it is not clear if these strict values also hold for larger systems and the thermodynamic limit. Besides, the work by Irish et al ${ }^{27]}$ is exclusively concerned with the fixed density transition occurring at $t \approx|\Delta|$, whereas a polariton SF may exist also for $t<|\Delta|$ if density fluctuations are allowed (generic transition). The spectrum in Fig. 13, b) is for such a set of parameters.

Again pertaining to the fixed density case, the MI state is supposed to be of excitonic nature for $\Delta / g<-1, t<|\Delta|$, and of polaritonic nature for $|\Delta| / g<1$ and small enough $t / g$ respectively $t /|\Delta|{ }^{[27}$ The former case is depicted in Fig. 13 (a), whereas the latter corresponds to the $\Delta=0$ results reported in Fig. 5 .

For $\Delta \gg g$, photon excitations are always lower in energy, and the effective interaction approaches zero. As a result, MI regions are very small or nonexistent, and the photonic SF state is similar to that of the Bose-Hubbard model in the limit of large $t / U$.24

Here we consider $\Delta / g= \pm 2$ for comparison to previous calculations of the spectra in the Mott phase.24 (Note that the rotating wave approximation formally requires $|\Delta| \ll$ $\epsilon, \omega_{0} \stackrel{68}{6}$ ) These correspond to effective repulsions $U_{\text {eff }} / g=$ 
0.096 (for $\Delta / g=2$ ) respectively $U_{\text {eff }} / g=2.096$ (for $\Delta / g=-2$ ), in excellent agreement with the width of the $n_{\mathrm{p}}=1$ Mott lobes for the same parameters. ${ }^{24}$

Our results in Fig. 13 show that again the spectra are dominated by the generic features of the MI and the SF. However, the detuning in the present case changes the ratio of the bandwidths of particle and hole bands $W_{\mathrm{p}} / W_{\mathrm{h}}$ in the Mott state.24 While for $\Delta=0, W_{\mathrm{p}} / W_{\mathrm{h}} \approx 3$, we find $W_{\mathrm{p}} / W_{\mathrm{h}} \approx 2$ (similar to the result for the Bose-Hubbard model) for $\Delta / g=2$ and $W_{\mathrm{p}} / W_{\mathrm{h}} \approx 7$ for $\Delta / g=-2$. The incoherent features observed for $\Delta / g=-2$ in Ref. 24 are not seen here. As mentioned before, the energy of the upper polariton modes (not shown) increases for $\Delta \neq 0,]^{7}$

In the SF, we find the expected gapless excitations, as well as gapped modes indicative of a correlated superfluid. Since for $\Delta / g=2, U_{\text {eff }}$ is very small, the Mott gap of the dispersive bands in Fig. 13.c) is also small $(0.057 g)$, but it is still larger than the temperature scale in our simulation $T / g=0.005$. We note that, within our resolution, the positive energy spectrum in Fig. 13 d) looks gapless, but not clearly linear. In this respect, the spectra for finite detuning resemble those obtained at high temperatures. Apart from this issue and a scaling of energies (due to the dependence of $U_{\text {eff }}$ on $\Delta$ ), the spectra obtained for $\Delta / g=-2$ are very similar to those for $\Delta=0$, whereas those for $\Delta / g=2$ resemble closely the results for the Bose-Hubbard model.

\section{Phase transition}

To end with, we present a scaling analysis for the generic phase transition. As pointed out by Fisher et al ${ }^{1}$ the scaling relation

$$
\rho_{\mathrm{s}}=L^{2-d-z} \widetilde{\rho}\left(\delta L^{1 / \nu}, \beta / L^{z}\right)
$$

should hold for the superfluid density across the MI-SF transition. Here $\nu$ is the critical exponent of the correlation length which is expected to diverge like $\xi \sim \delta^{-\nu}$, and $z$ is the dynamical critical exponent. The generic transition in the Bose-Hubbard model has mean-field exponents $z=2$ and $v=1 / z=1 / 22^{[12157 / 69}$ Recent field theory ${ }^{10}$ and strongcoupling results ${ }^{25}$ predict the same universality classes for the polariton model, in conflict with numerical results in two dimensions which suggest the absence of multicritical points. $\frac{53}{53}$

We test in 1D the scaling hypothesis Eq. (12) with $z=2$ and the hyperscaling relation $z=1 / \nu$, along the line $\mu / g=$ 0.4 where the generic transition is expected [see Fig. 11 b)].

To this end we keep the temperature constant at $\beta=L^{2} / 10$ and plot $\rho_{\mathrm{s}} L^{d+z-2}$ over $\left(t-t_{\mathrm{c}}\right) L^{1 / \nu}$ to obtain the universal function $\widetilde{\rho}$ (Fig. 14). Defining a cost function in the spirit of Ref. 70, allows us to evaluate the quality of the finite-sizescaling plot quantitatively.

We find the minimum of our cost function at $t_{\mathrm{c}}=$ $0.0626(1)$ and $\nu=0.50(2)$ when matching $\widetilde{\rho}$ close to the phase transition $\left[\left|\left(t-t_{c}\right) L^{1 / \nu}\right|<1\right.$ in Fig. 14]. We note that with the system sizes available, this result is not very stable. A fit in a larger region of $\left|\left(t-t_{c}\right) L^{1 / \nu}\right|$ provides a better data

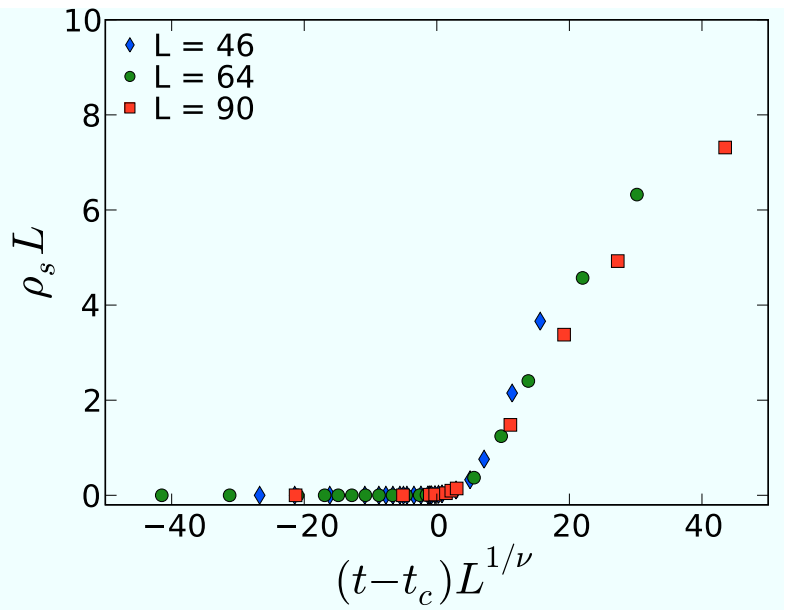

FIG. 14: (color online) Finite size scaling for the generic transition in the polariton model at $\mu / g=0.4$, testing the scaling hypothesis Eq. (12).

collapse overall (but worse close to the phase transition), with $\nu \approx 0.65$ and very slightly smaller $t_{\mathrm{c}}$.

A similar scaling with $z=1$ did not succeed, so that we conclude that the universality class of the generic transition in the polariton model is the same as that in the BoseHubbard model, despite the composite nature of the quasiparticles. This is consistent with recent field-theory and strongcoupling results $\frac{1025}{}$

An accurate scaling analysis for the fixed density transition through the lobe tip has been found to require much larger system sizes and is therefore not shown. In the 1D case considered, the shape of the lowest Mott lobe in 1D (see Fig. 1) suggests that the similarity to the Bose-Hubbard model holds also in this respect, i.e. a Kosterlitz-Thouless type phase transition.

\section{v. CONCLUSIONS}

We calculated the single-boson spectral function and the dynamic structure factor of the Bose-Hubbard model, and for a recently proposed model of itinerant polaritons in coupledcavity arrays. These models undergo a quantum phase transition from a Mott insulator to a superfluid state upon increasing the hopping integral of the bosons respectively photons with respect to the interaction. Results in one dimension, within and close to the Mott lobe with density one, have been obtained.

Despite the generally different nature of the conserved particles, the models exhibit very similar spectral properties, including gapped particle and hole bands in the Mott insulating phase, and Bogoliubov type excitations in the superfluid phase. Additional excitations related to the second branch of upper polariton states exist in the single-particle spectrum of the polariton model $[25$ but cancel out in the dynamic structure factor. In general, these features have high energy and very small spectral weight, so that for practical purposes the ex- 
citation spectra are qualitatively similar to the Bose-Hubbard model.

Correlation effects are particularly strong in the one dimensional case considered. Our results in the superfluid phase represent the first unbiased nonperturbative spectra for $A(k, \omega)$ in both models and for $S(k, \omega)$ in the polariton model (in both phases). Good qualitative agreement with recent analytical work on the two-dimensional Bose-Hubbard model was found, and we have compared our results in the superfluid phase to Bogoliubov theory. The limiting cases of the Mott insulator close to the atomic limit, as well as the weakly interacting superfluid are described quite well by analytical approximations, whereas in the phase transition region, our nonperturbative results show considerable deviation. Emerging particle-hole symmetry on approach of the multicritical lobe tip has been demonstrated for the polariton model.

For the polariton model, we have also explored the influence of detuning and finite temperature on the spectral properties, and have presented a scaling analysis to determine the universality class of the generic phase transition. Keeping in mind experimental realizations of coupled cavity arrays, interesting open issues for future work include the excitation spec- tra in the two-dimensional case (and comparison to analytical results ${ }^{25}$ ), the behavior of the sound velocity across the phase transition (also for the Bose-Hubbard model) and disorder.

The present work further highlights the fact that the physics of strongly correlated bosons as described by the BoseHubbard model may be observed in terms of optical models that, if realized, would have some distinct experimental advantages and further contain new degrees of freedom due to the mixed nature of the quasiparticles.

\section{Acknowledgments}

MH was supported by the FWF Schrödinger Fellowship No. J2583. PP acknowledges support from the FWF, projects P18551 and P18505. We made use of the ALPS library 4445 and the ALPS applications. ${ }^{[6]}$ We acknowledge fruitful discussions with F. Assaad, M. J. Bhaseen, J. Keeling, D. Khmelnitskii and P. B. Littlewood. We are grateful to H. Monien and D. Rossini for providing us with data for Figure 1 .
* Present address: OSRAM Opto Semiconductors, 93055 Regensburg, Germany

${ }^{1}$ M. P. A. Fisher, P. B. Weichman, G. Grinstein, and D. S. Fisher, Phys. Rev. B 40, 546 (1989).

2 M. Greiner, O. Mandel, T. Esslinger, T. W. Hänsch, and I. Bloch, Nature 415, 39 (2002).

3 K. M. Birnbaum et al., Nature 436, 87 (2005).

${ }^{4}$ F. Brennecke et al., Nature 450, 268 (2007).

5 J. M. Fink et al., Nature 454, 315 (2008).

6 M. J. Bhaseen, M. Hohenadler, A. O. Silver, and B. D. Simons, Phys. Rev. Lett. 102, 135301 (2009).

7 A. D. Greentree, C. Tahan, J. H. Cole, and L. C. L. Hollenberg, Nat. Phys. 2, 856 (2006).

${ }^{8}$ M. J. Hartmann, F. G. S. L. Brandão, and M. B. Plenio, Nat. Phys. 2, 849 (2006).

9 M. Hartmann, F. Brandao, and M. Plenio, Laser Photonics Rev. 2, 527 (2008).

10 J. Koch and K. Le Hur, Phys. Rev. A 80, 023811 (2009).

11 W. Zwerger, J. Opt. B: Quantum Semiclass. Opt. 5, 9 (2003).

${ }^{12}$ K. Sheshadri, H. R. Krishnamurthy, R. Pandit, and T. V. Ramakrishnan, Europhys. Lett. 22, 257 (1993).

13 N. Elstner and H. Monien, Phys. Rev. B 59, 12184 (1999).

14 T. D. Kühner, S. R. White, and H. Monien, Phys. Rev. B 61, 12474 (2000).

15 R. Roth and K. Burnett, J. Phys. B 37, 3893 (2004).

${ }^{16}$ G. G. Batrouni, F. F. Assaad, R. T. Scalettar, and P. J. H. Denteneer, Phys. Rev. A 72, 031601(R) (2005).

17 K. Sengupta and N. Dupuis, Phys. Rev. A 71, 033629 (2005).

18 W. Koller and N. Dupuis, J. Phys.: Condens. Matter 18, 9525 (2006).

19 S. D. Huber, E. Altman, H. P. Büchler, and G. Blatter, Phys. Rev. B 75, 085106 (2007).

${ }^{20}$ B. Capogrosso-Sansone, S. G. Söyler, N. Prokof'ev, and B. Svistunov, Phys. Rev. A 77, 015602 (2008).

21 B. Capogrosso-Sansone, N. V. Prokof'ev, and B. V. Svistunov,
Phys. Rev. B 75, 134302 (2007).

${ }^{22}$ M. Ohliger and A. Pelster, arXiv:0810:4399v1 (2008).

23 C. Menotti and N. Trivedi, Phys. Rev. B 77, 235120 (2008).

${ }^{24}$ M. Aichhorn, M. Hohenadler, C. Tahan, and P. B. Littlewood, Phys. Rev. Lett. 100, 216401 (2008).

${ }^{25}$ S. Schmidt and G. Blatter, Phys. Rev. Lett. 103, 086403 (2009).

26 A. Griffin, Excitations in a Bose-Condensed Liquid (Cambridge University Press, 1993).

${ }^{27}$ E. K. Irish, C. D. Ogden, and M. S. Kim, Phys. Rev. A 77, 033801 (2008).

28 S. C. Lei and R. K. Lee, Phys. Rev. A 77, 033827 (2008).

${ }^{29}$ E. K. Irish, arXiv:0903.3380 (2009).

${ }^{30}$ J. Cho, D. G. Angelakis, and S. Bose, Phys. Rev. Lett. 101, 246809 (2008).

31 J. K. Freericks and H. Monien, Phys. Rev. B 53, 2691 (1996).

32 L. Pollet, S. M. A. Rombouts, and P. J. H. Denteneer, Phys. Rev. Lett. 93, 210401 (2004).

33 O. F. Syljuåsen and A. W. Sandvik, Phys. Rev. E 66, 046701 (2002).

${ }^{34}$ H. Shi and A. Griffin, Phys. Rep. 304, 1 (1998).

35 D. G. Angelakis, M. F. Santos, and S. Bose, Phys. Rev. A 76, 031805(R) (2007).

${ }^{36}$ M. J. Hartmann and M. B. Plenio, Phys. Rev. Lett. 100, 070602 (2008).

37 E. T. Jaynes and F. W. Cummings, Proc. IEEE 51, 89 (1963).

38 M. I. Makin, J. H. Cole, C. Tahan, L. C. L. Hollenberg, and A. D. Greentree, Phys. Rev. A 77, 053819 (2008).

39 D. Rossini and R. Fazio, Phys. Rev. Lett. 99, 186401 (2007).

40 D. Rossini, R. Fazio, and G. Santoro, EPL 83, 47011 (2008).

${ }^{41}$ J. Koch, T. M. Yu, J. Gambetta, A. A. Houck, D. I. Schuster, J. Majer, A. Blais, M. H. Devoret, S. M. Girvin, and R. J. Schoelkopf, Phys. Rev. A 76, 042319 (2007).

${ }^{42}$ H. G. Evertz, G. Lana, and M. Marcu, Phys. Rev. Lett. 70, 875 (1993).

43 H. G. Evertz, Adv. Phys. 52, 1 (2003). 
44 A. Albuquerque et al., J. Magn. Magn. Mater. 310, 1187 (2007).

${ }^{45}$ M. Troyer, B. Ammon, and E. Heeb, Lect. Notes Comput. Sci. 1505, 502 (1998).

${ }^{46}$ F. Alet, S. Wessel, and M. Troyer, Phys. Rev. E 71, 036706 (2005).

47 A. Sandvik and J. Kurkijärvi, Phys. Rev. B 43, 5950 (1991).

48 A. Dorneich and M. Troyer, Phys. Rev. E 64, 066701 (2001).

49 A. W. Sandvik, R. R. P. Singh, and D. K. Campbell, Phys. Rev. B 56, 14510 (1997).

${ }^{50}$ F. Michel and H. G. Evertz, arXiv.org/0705.0799 (2007).

51 E. L. Pollock and D. M. Ceperley, Phys. Rev. B 36, 8343 (1987).

${ }^{52}$ N. V. Prokofev and B. V. Svistunov, Phys. Rev. B 61, 11282 (2000).

53 J. Zhao, A. W. Sandvik, and K. Ueda, arXiv:0806.3603 1 (2008).

54 J. K. Freericks and H. Monien, Europhys. Lett. 26, 545 (1994).

55 T. D. Kühner and H. Monien, Phys. Rev. B 58, R14741 (1998).

${ }^{56}$ F. Gerbier, Phys. Rev. Lett. 99, 120405 (2007).

57 F. Alet and E. S. Sorensen, Phys. Rev. B 70, 024513 (2004).

${ }^{58}$ B. Paredes et al., Nature 429, 277 (2004).

59 N. Fabbri et al., Phys. Rev. A 79, 043623 (2009).
${ }^{60}$ N. N. Bogoliubov, J. Phys. (USSR) 11, 23 (1947).

61 J. M. Vogels, K. Xu, C. Raman, J. R. Abo-Shaeer, and W. Ketterle, Phys. Rev. Lett. 88, 060402 (2002).

${ }^{62}$ A. M. Rey et al., J. Phys. B 36, 825 (2003).

${ }^{63}$ D. van Oosten, P. van der Straten, and H. T. C. Stoof, Phys. Rev. A 63, 053601 (2001).

${ }^{64}$ X. Lu, J. Li, and Y. Yu, Phys. Rev. A 73, 043607 (2006).

65 A. B. Bhattacherjee, J. Phys. B 40, 143 (2007).

${ }^{66}$ D. B. M. Dickerscheid, D. van Oosten, P. J. H. Denteneer, and H. T. C. Stoof, Phys. Rev. A 68, 043623 (2003).

${ }^{67}$ L. I. Plimak, M. K. Olsen, and M. Fleischhauer, Phys. Rev. A 70, 013611 (2004).

${ }^{68}$ N. B. Narozhny, J. J. Sanchez-Mondragon, and J. H. Eberly, Phys. Rev. A 23, 236 (1981).

${ }^{69}$ G. G. Batrouni, R. T. Scalettar, and G. T. Zimanyi, Phys. Rev. Lett. 65, 1765 (1990).

${ }^{70}$ K. Harada and N. Kawashima, J. Phys. Soc. Jpn. 67, 2768 (1998). 